\title{
Temperature Dependence of the Partially Localized State in a 2D Molecular Nanoporous Network
}

\author{
Ignacio Piquero-Zulaica ${ }^{1, *}$, Sylwia Nowakowska ${ }^{2}$, J.Enrique Ortega ${ }^{1,3,4}$, Meike Stöhr ${ }^{5}$, Lutz \\ H. Gade ${ }^{6}$, Thomas A. Jung ${ }^{7}$, Jorge Lobo-Checa ${ }^{8,9, *}$ \\ ${ }^{1}$ Centro de Física de Materiales (CSIC/UPV-EHU) -Materials Physics Center, Manuel Lardizabal 5, \\ 20018 San Sebastián, Spain. \\ ${ }^{2}$ Department of Physics, University of Basel, Klingelbergstrasse 82, 4056 Basel, Switzerland. \\ ${ }^{3}$ Donostia International Physics Center (DIPC), Manuel Lardizabal 4, 20018 San Sebastián, Spain. \\ ${ }^{4}$ Departamento Física Aplicada I, Universidad del País Vasco, 20018 San Sebastián, Spain. \\ ${ }^{5}$ Zernike Institute for Advanced Materials, University of Groningen, Nijenborgh 4, 9747 AG \\ Groningen, The Netherlands. \\ ${ }^{6}$ Anorganisch-Chemisches Institut, Universität Heidelberg, Im Neuenheimer Feld 270, 69120 \\ Heidelberg, Germany. \\ ${ }^{7}$ Laboratory for Micro- and Nanotechnology, Paul Scherrer Institute, 5232 Villigen PSI, Switzerland. \\ ${ }^{8}$ Instituto de Ciencia de Materiales de Aragón (ICMA), CSIC-Universidad de Zaragoza, E-50009 \\ Zaragoza, Spain. \\ ${ }^{9}$ Departamento de Física de la Materia Condensada, Universidad de Zaragoza, E-50009 Zaragoza, \\ Spain. \\ * e-mail: I.P.-Z. ipiquerozulaica@gmail.com, J.L.-C. jorge.lobo@csic.es
}

\begin{abstract}
Two-dimensional organic and metal-organic nanoporous networks can scatter surface electrons, leading to their partial localization. Such quantum states are related to intrinsic surface states of the substrate material. We further corroborate this relation by studying the thermally induced energy shifts of the electronic band stemming from coupled quantum states hosted in a metalorganic array formed by a perylene derivative on $\mathrm{Cu}(111)$. We observe by angle-resolved photoemission spectroscopy (ARPES), that both, the Shockley and the partially localized states, shift by the same amount to higher binding energies upon decreasing the sample temperature, providing evidence of their common origin. Our experimental approach and results further support the use of surface states for modelling these systems, which are expected to provide new insight into the physics concerning partially confined electronic states: scattering processes, potential barrier strengths, excited state lifetimes or the influence of guest molecules.
\end{abstract}

Keywords: Metal-organic coordination, self-assembly, nanoarchitectures, confinement, angleresolved photoemission spectroscopy, scanning tunneling spectroscopy.

Abbreviations: DPDI (4,9-diaminoperylene quinone-3,10-diimine), ARPES (angle-resolved photoemission spectroscopy), STM (scanning tunneling microscopy), UHV (ultrahigh vacuum), EDC (energy distribution curve), SS (Shockley state), PLS (partially localized state) 


\section{Introduction}

Surface states emerge as additional solutions of the Schrödinger equation due to the sudden termination of the 3D periodic bulk. They are located in the projected bulk band gaps and localized at the outermost atomic layers. Surface states provide ideal systems to study low dimensional electronic properties and are extremely sensitive to surface defects such as adsorbates or steps [1$6]$.

Noble metals exhibit such surface states, generally referred to as Shockley states [7], which generate a quasi-two-dimensional, nearly-free electron gas from $s p$-derived states in the direction parallel to the surface plane, but are confined by the vacuum barrier and the bottom of the projected bulk band gap perpendicular to it [8]. In particular, (111) terminated crystals accommodate their Shockley states at the center of the surface Brillouin zone with well-defined effective mass and parabolic energy dispersion relations $[9,10]$. The energy onset of these Shockley states is related to the position of the projected bulk band gaps, which are highly sensitive to temperature-induced variations of the lattice constant, causing the supported Shockley state to shift in energy [11].

Previous works have already demonstrated that Shockley electrons can be confined parallel to the surface by artificially built nanoscale assemblies [12-15]. A similar electron localization has been shown in two-dimensional (2D) organic nanoporous networks [4,16-20]. Owing to their periodicity, these networks can be considered as arrays of quantum boxes. However, such confinements are often imperfect, allowing for some degree of coupling between neighboring pores that gives rise to shallow dispersive electronic bands [16]. The resulting electronic band has been assigned to stem from the pristine surface state $[4,16]$, but direct experimental evidence to this assignment is desirable to further corroborate it.

In this letter, we study the temperature dependence of the electronic structure which arises from the interaction of the Cu-coordinated triply dehydrogenated perylene derivative DPDI (4,9diaminoperylene quinone-3,10-diimine) porous network on $\mathrm{Cu}(111)$ using angle-resolved photoemission spectroscopy (ARPES). In dependence of both the sample temperature and the network coverage, we investigate the evolution of the Shockley and the partially localized states. We find that both electronic states experience identical energy shifts upon varying the sample temperature. This provides evidence that the partially localized state indeed originates from the Shockley state and appears modified by the potential of the molecular network.

\section{Experimental details}

ARPES measurements were performed in UHV (ultrahigh vacuum) conditions (base pressure of $1 \times 10^{-10}$ mbar) with a lab-based experimental setup equipped with a display-type hemispherical electron analyzer (SPECS Phoibos 150), an energy/angle resolution of $40 \mathrm{meV} / 0.1^{\circ}$ and a monochromatized Helium I ( $\mathrm{hv}=21.2 \mathrm{eV})$ source. Variable temperature measurements were 
performed using a closed-cycle He cryogenic manipulator with a controlled temperature range between $350 \mathrm{~K}$ and $35 \mathrm{~K}$.

The STM (scanning tunneling microscopy) measurements were carried out at both low temperature (Omicron Nanotechnology $\mathrm{GmbH}$ with Nanonis SPM control system) and room temperature (home built STM). The bias voltages given in the manuscript refer to a grounded tip. The measurements were performed with Pt-Ir tips $(90 \% \mathrm{Pt}, 10 \% \mathrm{Ir})$ prepared by mechanical cutting and followed by in situ sputtering with Ar+ ions and controlled indentation in the bare $\mathrm{Cu}(111)$ substrate. STM data were acquired in constant current mode and were processed with the WSxM software [21].

The $\mathrm{Cu}(111)$ surface was cleaned by repeated cycles of $\mathrm{Ne}^{+}$sputtering at energies of $1.0 \mathrm{keV}$, followed by electron bombardment annealing at $800 \mathrm{~K}$. This resulted in a clean and well-ordered surface as verified by the spectral linewidth of the $\mathrm{Cu}(111)$ Shockley state [9].

DPDI [22-24] was sublimed from a Knudsen cell ( $515 \mathrm{~K}$ ) onto $\mathrm{Cu}(111)$ held at RT in a gradient coverage geometry from 0 to 1 monolayer (ML), as shown in Fig. 1(a). $1 \mathrm{ML}$ is defined as saturating the surface with molecules in the closed-packed assembly [24]. Prior to deposition, the molecular rate was monitored by a quartz crystal microbalance. Subsequent to deposition of the well-defined coverage gradient of DPDI, the sample was annealed to $550 \mathrm{~K}$ [25] such that a homogeneous Cu-coordinated metal-organic network was generated in the lower coverage regions, similar to the model depicted in Fig. 1(b). This nanoporous arrangement led to a sharp and intense signal visible in the ARPES channel plate detector close to the Fermi energy [16].

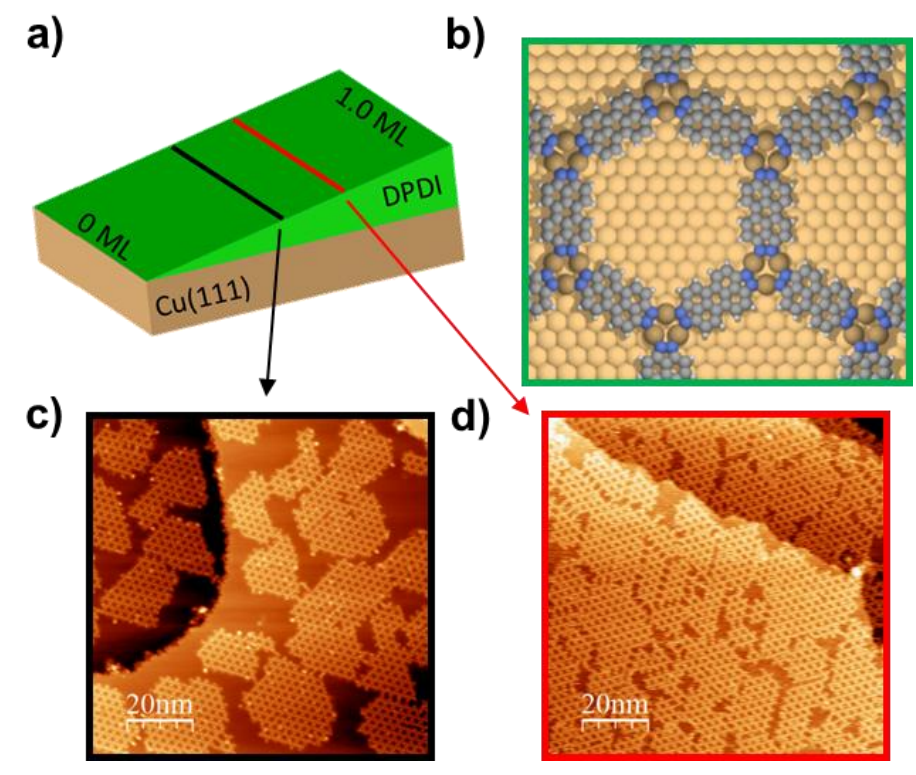

Figure 1. A variable density quantum box array realized through a coverage gradient geometry. (a) Schematic view of the DPDI deposition gradient performed on $\mathrm{Cu}(111)$. (b) Structural model for the Cucoordinated 3deh-DPDI nanoporous network forming a $\mathrm{p}(10 \times 10)$ superstructure on $\mathrm{Cu}(111)$ (adapted from 
Ref. [23]). (c, d) STM images for $0.45 \mathrm{ML}$ (left) (4 K, -0.8 V, $5 \mathrm{pA}$ ) and $0.65 \mathrm{ML}$ (right) (297 K, $2 \mathrm{~V}, 6 \mathrm{pA})$ network coverage. The network structure is maintained throughout the coverage gradient up to $\sim 0.73 \mathrm{ML}$.

The coverage gradient allowed us to study different network density regions within the same sample preparation (see Fig. 1(a)). In essence, well-formed molecular network islands progressively colonize the surface as the molecular coverage is increased, as shown by the STM images of Figs. 1(c) and (d) (0.45ML and 0.65 ML, respectively) [23]. Above the critical coverage of $\sim 0.73 \mathrm{ML}$, the porous network collapses into a close-packed assembly [24].

\section{Results and discussion}

ARPES spectral functions close to the Fermi level were systematically acquired as function of sample temperature (from $315 \mathrm{~K}$ to $136 \mathrm{~K}$ ) for two coverage regions: the first, where the molecular network completely covered the surface $(\sim 0.75 \mathrm{ML})$ and the second, where it coexisted with molecule-free areas ( $0.50 \mathrm{ML}$ ) (close to the coverage of Fig. 1(c)). The 3deh-DPDI molecules on the surface act as scattering centers for the surface electrons [26], generating confining potentials at the pores. For this network, the charge transfer to the molecules occurs only through the coordinating $\mathrm{Cu}$ adatoms, accordingly reducing the molecule-substrate interactions [23].

Figure 2(a) shows the ARPES spectral function corresponding to the temperature extremes for $\sim 0.50 \mathrm{ML}$ DPDI. The intensity distributions $\mathrm{I}\left(\mathrm{E}-\mathrm{E}_{\mathrm{F}}, \mathrm{k}_{\mathrm{II}}\right)$ reflect a coexistence of the Cu Shockley state (SS) band originating from the molecule-free areas and the partially localized state (PLS) band arising from the coupling between pores, in agreement with Ref. [16]. While the former has an upward parabolic dispersion [9], the latter exhibits a lower binding energy at $\bar{\Gamma}$ due to partial confinement effects together with an apparent increase of the effective mass [27]. Note that there is a slight energy offset $(\sim 40 \mathrm{meV})$ of the SS for the presented lower coverage case with respect to the pristine SS $[5,9]$. This is probably caused by scattering at the edges of the randomly distributed network islands, which cover $\sim 70 \%$ of the available surface (cf. Fig. 1.(c)). For such a coverage we note that the relative intensities of the states is not even. The high defect concentration and the small average island size effectively decrease the PLS signal with respect to the SS [16].

As the temperature of the sample decreases from $315 \mathrm{~K}$ to $136 \mathrm{~K}$, both electronic bands of Fig. 2.(a) become better defined and gain amplitude $[9,28]$. As expected, the bottom of the $\mathrm{Cu}$ Shockley state band shifts towards higher binding energy [11,29], but it is difficult to discern the behavior of the PLS band from the raw data. To follow its evolution, the energy distribution curves (EDC) of the ARPES spectral functions at $\bar{\Gamma}\left(k_{\mid l}=0\right)$ are represented in Fig. $2(\mathbf{b})$ for both end temperatures, red being the higher temperature spectrum and blue the lower one. We observed that the well-known Shockley state band minimum (peak closest to $E_{i}=-0.40 \mathrm{meV}$ ) was shifted away from the Fermi energy by $\sim 30 \mathrm{meV}$ as the sample was cooled down. The same trend was observed on the shoulder feature located in the proximity of $E_{i}=-0.25 \mathrm{eV}$. This peak corresponds to the PLS and is in part masked by its proximity to the SS. For a better visualization of the thermal energy shift of this state, it is best to study the higher coverage regions which lack the molecule- 
free areas, for which the Shockley state is quenched. This case is shown in Fig. 2(c), corresponding to $0.75 \mathrm{ML}$ and exhibiting only bands attributable to the porous network. Once more a clear energy shift of the band minimum and a sharpening of the features were observed as the temperature was lowered. The comparison between the temperature dependent EDCs, represented in Fig. 2(d), shows that the PLS also shifts around $30 \mathrm{meV}$ to higher binding energies as the sample is cooled down.

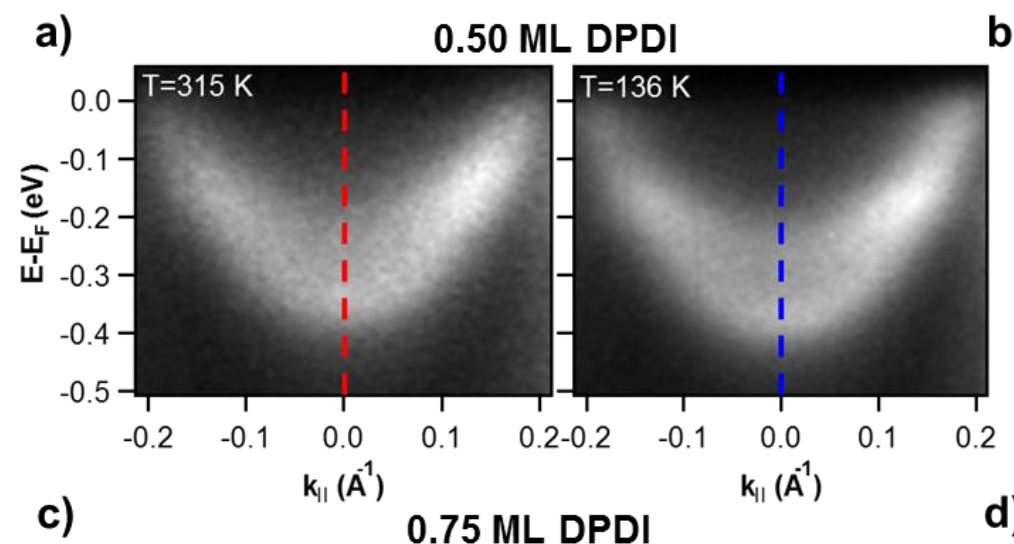

b)

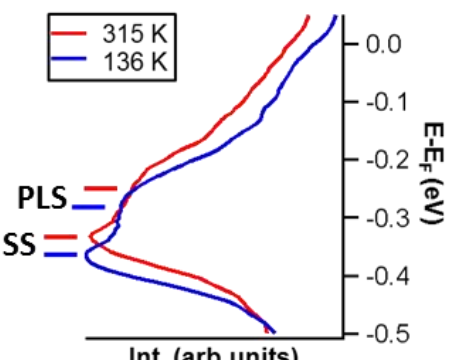

d)
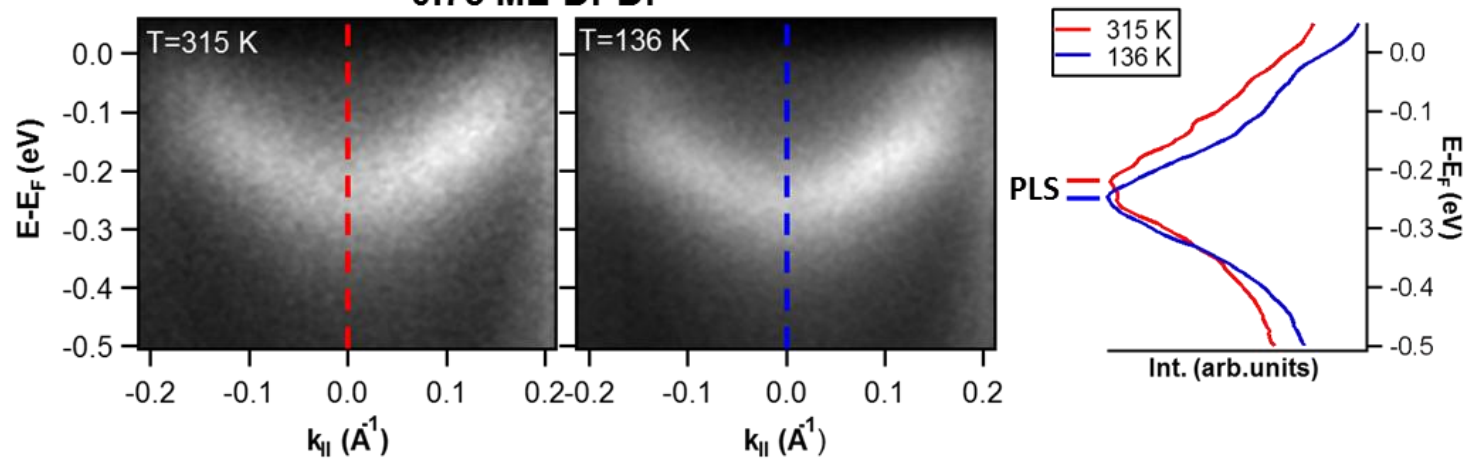

Figure 2. Temperature dependence of the network partially localized state band and Cu Shockley state band at the two extreme temperatures studied. In contrast to Ref. [16], raw ARPES data are presented here to directly compare the resulting energy distribution curves (EDCs). (a) ARPES experimental spectral function acquired at $315 \mathrm{~K}$ and $136 \mathrm{~K}$ for the coverage of $0.50 \mathrm{ML}$, in which regions of the network and molecule-free areas coexist. (b) Smoothened, normal emission ( $\left.k_{\| \prime}=0\right)$ EDCs for both temperatures in (a). The horizontal lines mark the energy positions of the Cu Shockley state (SS) and the partially localized state (PLS) band minima obtained from a two component Lorentzian fit. (c) ARPES experimental spectral function acquired at $315 \mathrm{~K}$ and $136 \mathrm{~K}$ for the coverage of $0.75 \mathrm{ML}$ featuring the surface completely covered by the network. (d) Smoothened, normal emission ( $\left.k_{\|}=0\right)$ EDCs for both temperatures in (c) with horizontal lines indicating the energy positions of the PLS band minimum.

The nature of the energy shift observed for the PLS is still undefined at this stage and we thus followed the temperature transition at intermediate points. In this regard, the Shockley state of $\mathrm{Cu}(111)$ has been shown to linearly shift with temperature, with a slope of $(1.8 \pm 0.1) \times 10^{-4} \mathrm{eV} / \mathrm{K}$ [11]. For the nanoporous network, EDC waterfall plots at normal emission for the studied 
temperature range are shown in Figs. 3(a) and (b) corresponding to full network coverage (0.75 $\mathrm{ML})$ and to coexisting regions of molecular network and molecule-free areas $(0.50 \mathrm{ML})$. We observed that the energy position of the band minimum of the PLS as well as the SS shifted in a linear, progressive way as the temperature was varied. There were no abrupt energy changes of the features, which sharpened up as the temperature was lowered.

a)

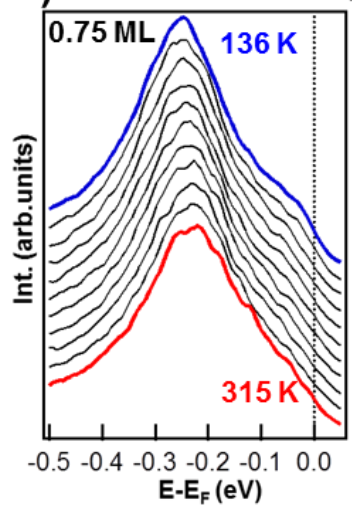

b)

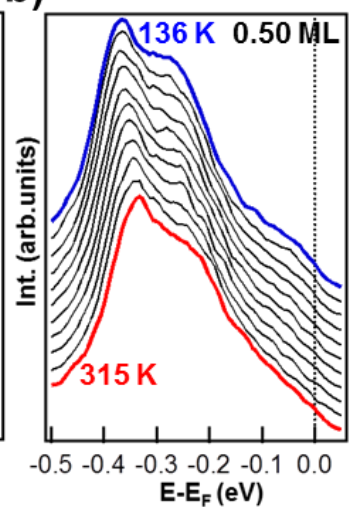

c)

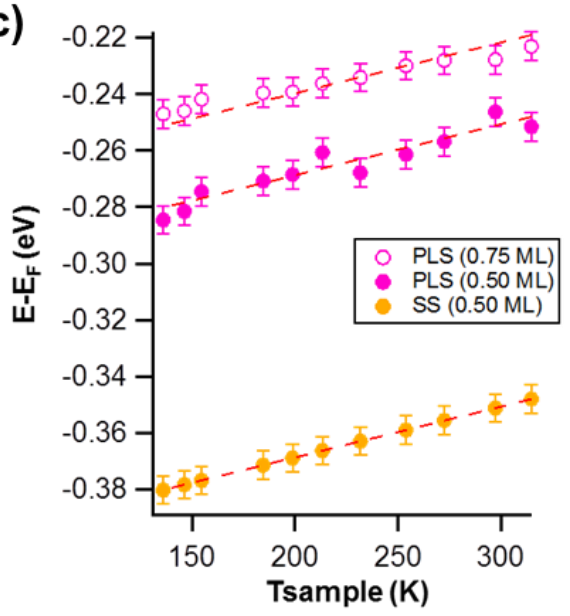

Figure 3. Full range temperature dependence of the partially localized state (PLS) and Cu Shockley state (SS). Normal emission EDC temperature waterfalls for $0.75 \mathrm{ML}$ (a) and $0.50 \mathrm{ML}$ (b) coverages. A clear trend towards higher binding energy and a sharpening of the features is observed as the temperature is lowered. (c) Temperature variation of the energy position of the PLS and SS band minima as extracted from the fit for each spectral line represented in the waterfall. The fit was carried out using one $(0.75 \mathrm{ML})$ or two $(0.50 \mathrm{ML})$ Lorentzian components convoluted with a linear background. The dashed lines correspond to variation rates with a common slope for the three components, all of which agree with the Shockley state variation reported in Ref. [11].

A quantitative analysis of the temperature dependence of these states was achieved by Lorentzian fitting and convolution with a linear background from the spectra in Figs. 3(a) and (b). Fig. 3(c) summarizes the results from such analysis and shows the variation of the initial state energy with sample temperature. As expected, the Cu SS band (yellow dots) follows the red dashed line which accounts for a rate of $(1.8 \pm 0.1) \times 10^{-4} \mathrm{eV} / \mathrm{K}$, in agreement with Paniago et al. [11], resulting in an increase of $32 \pm 2 \mathrm{meV}$ in binding energy for the investigated temperature range. The other two sets of magenta points in Fig. 3(c) correspond to the PLS for the $0.50 \mathrm{ML}$ (full circles) and $0.75 \mathrm{ML}$ (open circles) and they differ in energy by $\sim 30 \mathrm{meV}$. We attribute this offset to a slight excess of molecular coverage [24]. For $0.5 \mathrm{ML}$ the PLS is shifted $33 \pm 5 \mathrm{meV}$ within the probed temperature range, which agrees with the variation of the SS, while a slightly smaller shift of $26 \pm 5$ meV was measured for $0.75 \mathrm{ML}$. This is still consistent with the SS behavior within the experimental error and the deviation can be again related to the uncontrolled structural changes arising from excess molecular coverage. As the temperature dependence is unique for each of the electronic states in $\mathrm{Cu}(111)[29,30]$, the demonstration that the PLS associated with the nanoporous network exhibits 
an identical temperature variation of its energy to the surface state of the clean $\mathrm{Cu}(111)$ substrate, supports that both have the same Shockley nature.

Hence, upon the network formation, the surface state electrons are subjected to a 2D finite periodic potential imposed by the network structure. The network thus alters the Shockley state giving rise to the scattering phenomena capable of generating new band structures with different dispersion relations $[4,16]$. The experimental evidence presented in this study enforces the validity that electrons partially localized within the molecular pores originate from the Shockley state $[4,16-22,27]$. This rationale should be extensible to other substrates supporting surface states.

\section{Conclusions}

In conclusion, in this experimental study we validate the origin and nature of the partially localized state observed in a 2D metal-organic nanoporous network self-assembled on the $\mathrm{Cu}(111)$ surface. By studying the temperature dependence of two different coverage regimes of the network, we corroborate that the partially localized state existing in the pores of the network originates from the $\mathrm{Cu}(111)$ Shockley state. The energies of both states display the same temperature dependence, directly relating the PLS to the SS observed on the pristine metal surface. This experimental demonstration supports that the surface state electrons are affected by the periodic molecular potential of the network. Therefore, semi-empirical models can be confidently used to derive the observed electronic states and to model the surface potential created by molecular networks.

\section{Acknowledgements}

We acknowledge financial support from the Spanish Ministry of Economy (grant MAT2013-46593C6-4-P), the Basque Government (grant IT621-13), the Spanish Research Council (CSIC201560I022), the Swiss Nanoscience Institute (SNI), Swiss National Science Foundation (grants No. 200020-149713, 206021-121461), the Netherlands Organization for Scientific Research (NWO Vidi grant No. 700.10.424), the European Research Council (ERC-2012-StG 307760-SURFPRO) and the Paul Scherrer Institute.

\section{References}

[1] Forster, F., Bendounan, A., Ziroff, J., Reinert, F., 2006. Systematic studies on surface modifications by ARUPS on Shockley-type surface states. Surface Science 600, 3870-3874.

[2] Cottin, M.C., Bobisch, C.A., Schaffert, J., Jnawali, G., Sonntag, A., Bihlmayer, G., Möller, R., 2011. Anisotropic scattering of surface state electrons at a point defect on $\mathrm{Bi}(111)$. Applied Physics Letters 98, 022108.

[3] Scheybal, A., Müller, K., Bertschinger, R., Wahl, M., Bendounan, A., Aebi, P., Jung, T.A., 2009. Modification of the $\mathrm{Cu}(110)$ Shockley surface state by an adsorbed pentacene monolayer. Phys. Rev. B 79, 115406. 
[4] Kepčija, N., Huang, T.-J., Klappenberger, F., Barth, J.V., 2015. Quantum confinement in selfassembled two-dimensional nanoporous honeycomb networks at close-packed metal surfaces. The Journal of Chemical Physics 142, 101931.

[5] Baumberger, F., Greber, T., Delley, B., Osterwalder, J., 2002. Tailoring Confining Barriers for Surface States by Step Decoration: CO / Vicinal Cu(111). Physical Review Letters 88, 237601.

[6] Lobo, J., Michel, E.G., Bachmann, A.R., Speller, S., Kuntze, J., Ortega, J.E., 2004. Tuning the Surface State Dimensionality of Cu Nanostripes. Physical Review Letters 93, 137602.

[7] Shockley, W., 1939. On the Surface States Associated with a Periodic Potential. Physical Review 56, 317-323.

[8] Memmel, N., 1998. Monitoring and modifying properties of metal surfaces by electronic surface states. Surface Science Reports 32, 91-163.

[9] Reinert, F., Hüfner, S., 2005. Photoemission spectroscopy-from early days to recent applications. New Journal of Physics 7, 97-97.

[10]Kevan, S.D., Gaylord, R.H., 1987. High-resolution photoemission study of the electronic structure of the noble-metal (111) surfaces. Physical Review B 36, 5809-5818.

[11]Paniago, R., Matzdorf, R., Meister, G., Goldmann, A., 1995. Temperature dependence of Shockley-type surface energy bands on $\mathrm{Cu}(111), \mathrm{Ag}(111)$ and $\mathrm{Au}(111)$. Surface Science 336, 113-122.

[12]Crommie, M.F., Lutz, C.P., Eigler, D.M., 1993. Confinement of electrons to quantum corrals on a metal surface. Science 262, 218-220.

[13]Pennec, Y., Auwärter, W., Schiffrin, A., Weber-Bargioni, A., Riemann, A., Barth, J.V., 2007. Supramolecular gratings for tuneable confinement of electrons on metal surfaces. Nature Nanotechnology 2, 99-103.

[14]Niebergall, L., Rodary, G., Ding, H.F., Sander, D., Stepanyuk, V.S., Bruno, P., Kirschner, J., 2006. Electron confinement in hexagonal vacancy islands: Theory and experiment. Physical Review B 74, 195436.

[15]Mugarza, A., Ortega, J.E., 2003. Electronic states at vicinal surfaces. Journal of Physics: Condensed Matter 15, S3281-S3310.

[16]Lobo-Checa, J., Matena, M., Müller, K., Dil, J.H., Meier, F., Gade, L.H., Jung, T.A., Stöhr, M., 2009. Band Formation from Coupled Quantum Dots Formed by a Nanoporous Network on a Copper Surface. Science 325, 300-303.

[17]Klappenberger, F., Kühne, D., Krenner, W., Silanes, I., Arnau, A., García de Abajo, F.J., Klyatskaya, S., Ruben, M., Barth, J.V., 2009. Dichotomous Array of Chiral Quantum Corrals by a Self-Assembled Nanoporous Kagomé Network. Nano Letters 9, 3509-3514.

[18]Klappenberger, F., Kühne, D., Krenner, W., Silanes, I., Arnau, A., García de Abajo, F.J., Klyatskaya, S., Ruben, M., Barth, J.V., 2011. Tunable Quantum Dot Arrays Formed from SelfAssembled Metal-Organic Networks. Physical Review Letters 106, 026802.

[19]Wyrick, J., Kim, D.-H., Sun, D., Cheng, Z., Lu, W., Zhu, Y., Berland, K., Kim, Y.S., Rotenberg, E., Luo, M., Hyldgaard, P., Einstein, T.L., Bartels, L., 2011. Do Two-Dimensional “Noble Gas Atoms" Produce Molecular Honeycombs at a Metal Surface? Nano Letters 11, 2944-2948. 
[20]Wang, S., Wang, W., Tan, L.Z., Li, X.G., Shi, Z., Kuang, G., Liu, P.N., Louie, S.G., Lin, N., 2013. Tuning two-dimensional band structure of $\mathrm{Cu}(111)$ surface-state electrons that interplay with artificial supramolecular architectures. Physical Review B 88.

[21]Horcas, I., Fernández, R., Gómez-Rodríguez, J.M., Colchero, J., Gómez-Herrero, J., Baro, A.M., 2007. WSXM: A software for scanning probe microscopy and a tool for nanotechnology. Review of Scientific Instruments 78, 013705.

[22]Nowakowska, S., Wäckerlin, A., Kawai, S., Ivas, T., Nowakowski, J., Fatayer, S., Wäckerlin, C., Nijs, T., Meyer, E., Björk, J., Stöhr, M., Gade, L.H., Jung, T.A., 2015. Interplay of weak interactions in the atom-by-atom condensation of xenon within quantum boxes. Nature Communications 6, 6071.

[23] Matena, M., Björk, J., Wahl, M., Lee, T.-L., Zegenhagen, J., Gade, L.H., Jung, T.A., Persson, M., Stöhr, M., 2014. On-surface synthesis of a two-dimensional porous coordination network: Unraveling adsorbate interactions. Physical Review B 90, 125408.

[24] Stöhr, M., Wahl, M., Galka, C.H., Riehm, T., Jung, T.A., Gade, L.H., 2005. Controlling Molecular Assembly in Two Dimensions: The Concentration Dependence of Thermally Induced 2D Aggregation of Molecules on a Metal Surface. Angewandte Chemie International Edition 44, 7394-7398.

[25] Shchyrba, A., Wäckerlin, C., Nowakowski, J., Nowakowska, S., Björk, J., Fatayer, S., Girovsky, J., Nijs, T., Martens, S.C., Kleibert, A., Stöhr, M., Ballav, N., Jung, T.A., Gade, L.H., 2014. Controlling the Dimensionality of On-Surface Coordination Polymers via Endo- or Exoligation. Journal of the American Chemical Society 136, 9355-9363.

[26] Gross, L. et al, 2004. Scattering of Surface State Electrons at Large Organic Molecules. Phys. Rev. Lett. 93, 056103.

[27] Nowakowska S. et al., submitted.

[28] Paniago, R., Matzdorf, R., Meister, G., Goldmann, A., 1995. High-resolution photoemission study of the surface states near gG on $\mathrm{Cu}(111)$ and $\mathrm{Ag}(111)$. Surface Science 331-333, 12331237.

[29]Knapp, J.A., Himpsel, F.J., Williams, A.R., Eastman, D.E., 1979. Temperature dependence of bulk and surface energy bands in copper using angle-resolved photoemission. Physical Review B 19, 2844-2849.

[30] Knoesel, E., Hotzel, A., Wolf, M., 1998. Temperature dependence of surface state lifetimes, dephasing rates and binding energies on $\mathrm{Cu}(111)$ studied with time-resolved photoemission. Journal of Electron Spectroscopy and Related Phenomena 88-91, 577-584. 